\title{
Determining the Volume of Additive Solution and Residual Plasma in Whole Blood Filtered and Buffy Coat Processed Red Cell Concentrates
}

\author{
Andrew Jordan ${ }^{a, b}$ Jason P. Acker ${ }^{a, b}$ \\ a Department of Laboratory Medicine and Pathology, University of Alberta, Edmonton, AB, Canada; \\ ${ }^{b}$ Center for Innovation, Canadian Blood Services, Edmonton, AB, Canada
}

\section{Keywords}

RBC processing - Additive solution - SAGM .

Residual plasma - Blood storage - Buffy coat .

Whole blood filtration

\section{Summary}

Background: Residual plasma in transfused red cell concentrates (RCCs) has been associated with adverse transfusion outcomes. Despite this, there is no consensus on the standard procedure for measuring residual plasma volume. Methods: The volumes of residual plasma and additive solution were measured in RCCs processed using two separation methods: whole blood filtration (WBF) and buffy coat (BC)/RCC filtration. The concentration of mannitol and albumin in RCC components was measured using colorimetric assays. Mannitol concentration was used to calculate additive solution volume. Residual plasma volume was calculated using two methods. Results: Calculated RCC supernatant volumes were much lower in BC-processed components compared to WBF-processed components $(\mathrm{BC}=97 \pm 6 \mathrm{ml}, \mathrm{WBF}=109$ $\pm 4 \mathrm{ml} ; \mathrm{p}<0.05$ ). Calculated additive solution volumes were greater in WBF- than in BC-processed components $(B C=81 \pm 4 \mathrm{ml}, \mathrm{WBF}=105 \pm 2 \mathrm{ml} ; \mathrm{p}<0.05)$. Absolute residual plasma volume varied significantly based on the calculation method used. Conclusion: Disparity between plasma volume calculation methods was observed. Efforts should be made to standardize residual plasma volume measurement methods in order to accurately assess the impact of residual plasma on transfusion outcomes.

(c) 2015 S. Karger GmbH, Freiburg

\section{Introduction}

Following collection, whole blood (WB) is processed into transfusable components, which are then stored until required. During WB processing, red blood cells (RBCs) are separated from plasma and other WB components by centrifugation. Blood services utilize a number of different processing techniques and use different centrifugation settings to fractionate transfusable components. Using currently available techniques, it is not possible to ensure the complete separation of plasma from the RBCs. The volume of residual plasma remains with the $\mathrm{RBC}$ component throughout storage, and whether or not this has a potential impact on RBC quality and transfusion outcome has not been fully investigated. Previously, the volume of residual plasma in stored red cell concentrates (RCCs) has been calculated using the following equations [1]:

Residual plasma volume $(\mathrm{ml})=$ supernatant volume $(\mathrm{ml})$

- additive solution volume $(\mathrm{ml})$

(Eq. 1).

Supernatant volume $(\mathrm{ml})=$ total unit volume $(\mathrm{ml})$

$\times(1-$ hematocrit $)$

RCC components at Canadian Blood Services are stored in saline-adenine-glucose-mannitol (SAGM) additive solution. The additive solution volume in the collection sets is approximately 110 $\mathrm{ml}$. This is a nominal value for the additive solution, as the suppliers give a margin of error of 104.5-121 ml. We previously attempted to calculate the volume of residual plasma in Canadian Blood Services RCC products by applying Eq. 1 and Eq. 2 to a large quality control database [2]. Using an expected additive solution volume of $110 \mathrm{ml}$, we observed a high frequency of negative values for residual plasma volume and significantly lower values in com-

\section{KARGER \\ Fax +497614520714

\section{() 2016 S. Karger GmbH, Freiburg}

$1660-3796 / 16 / 0432-0133 \$ 39.50 / 0$ 


\section{Whole Blood Filtered}

Fig. 1. WBF- and BC-processing methods. The sequence for sampling the WBF- and BC-RCCs for residual plasma and additive solution testing was as follows: 1) albumin samples were taken from plasma components; 2) mannitol samples were taken from the SAGM component prior its addition to the RBCs; 3) mannitol and albumin samples were taken from LR-RCC components following mixing with SAGM; 4) SAGM was drained through leukoreduction filter and mixed with RBCs, the SAGM-RBCs were passed back through filter into SAGM bag and mannitol and albumin samples were then taken from LR-RCC component following leukoreduction. $\mathrm{LR}=$ Leukoreduced.

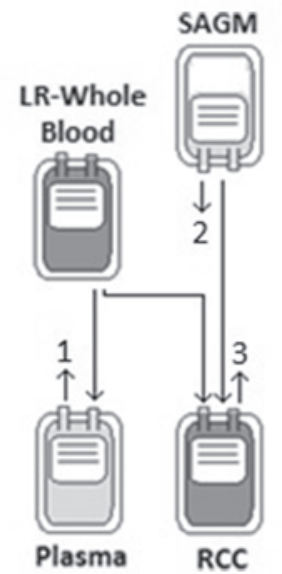

\section{Buffy-Coat Processing}

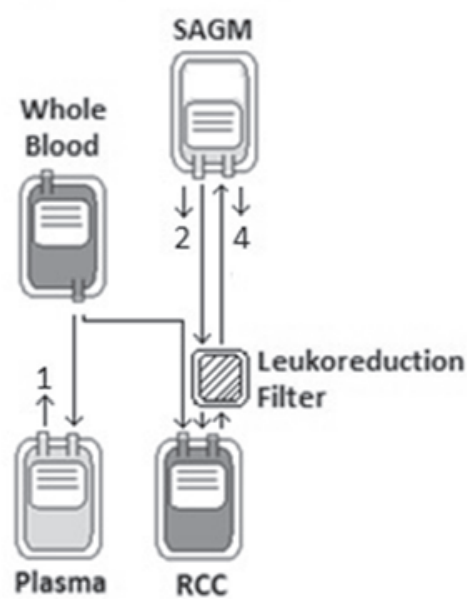

ponents processed using the buffy coat (BC) method. This led us to speculate that less than $110 \mathrm{ml}$ of additive solution is delivered to the RBCs during processing and that this value is affected by processing method. To investigate this further, we set out to accurately measure the volume of SAGM and residual plasma in whole bloodfiltered (WBF) and BC-processed RCC components.

\section{Materials and Methods}

\section{Whole Blood Collection and Processing}

WB was collected from 9 donors using Macopharma WBF collection sets (DQE 7291 LX Leucoflex MTL1 Quadruple Top and Top System, CPD/SAGM $500 \mathrm{ml}$; MacoPharma, Tourcoing, France) and from 9 donors using Macopharma BC collection sets (LQT 7291 LX Leucoflex LCR-Diamond Quadruple Bottom and Top System, CPD/SAGM $500 \mathrm{ml}$; Macopharma). The blood units were processed according to Canadian Blood Services standard operating procedures, as previously described [3]. Briefly; WBF units are leukoreduced then centrifuged at $4,552 \times g$ for 6 min to produce one unit of RCC and a unit of plasma. BC units are centrifuged prior to leukoreduction at $3,493 \times g$ for 11 min, yielding a unit of buffy coat in addition to the plasma and RCC. At given points throughout processing, samples were taken for mannitol and albumin measurements (fig. 1).

\section{Component Characteristics}

Total unit volume was calculated by dividing the tare weight $(\mathrm{g})$ of the component by $1.06 \mathrm{~g} / \mathrm{l}$ (specific gravity of WB at a standard hematocrit). Hematocrit was measured using hematology analyzer (Coulter AcT 8 Hematology Analyzer; Beckman Coulter, Brea, CA, USA).

\section{Mannitol Measurements}

Mannitol concentration was measured using a colorimetric assay (D-Mannitol Colorimetric Assay Kit; Sigma-Aldrich, St. Louis, MO, USA). For both WBF- and BC-processed units, a sample of SAGM was taken from the SAGM container before mixing with the RBCs to provide a baseline SAGM mannitol concentration. For WBF-processed units, samples were taken from the RCC container after the SAGM had been thoroughly mixed with the RBCs. For BCprocessed units, samples were taken from the RCC storage container after the SAGM had been mixed with the RBCs and the SAGM-RBC mixture had passed through the leukoreduction filter. RCC samples were centrifuged at 2,200 $\times g$ for 10 min to separate the RBCs and supernatant. $25 \mu \mathrm{l}$ of supernatant was removed and diluted in an equal volume of mannitol assay buffer. Samples and standards were added to reaction mixtures in a 96-well plate and incubated for
Table 1. RCC unit volumes and hematocrits for WBF- and BC-processed components $^{\mathrm{a}}$

\begin{tabular}{lllc|}
\hline & $\begin{array}{l}\text { Total unit } \\
\text { volume, } \mathrm{ml}\end{array}$ & Hematocrit, \% & $\begin{array}{c}\text { Supernatant } \\
\text { volume, } \mathrm{ml}\end{array}$ \\
\hline $\mathrm{WBF}(\mathrm{n}=9)$ & $305 \pm 13$ & $65 \pm 2$ & $109 \pm 4$ \\
$\mathrm{BC}(\mathrm{n}=9)$ & $260 \pm 11$ & $63 \pm 3$ & $97 \pm 6$ \\
\hline
\end{tabular}

aTotal unit volume was calculated by dividing unit tare weight $(\mathrm{g})$ by $1.06 \mathrm{~g} / \mathrm{l}$. Supernatant volumes were calculated using total unit volume and hematocrit (Eq. 2). Values shown are mean \pm SD.

$20 \mathrm{~min}$ at $37^{\circ} \mathrm{C}$. Absorbance was read at $450 \mathrm{~nm}$ using a spectrophotometric plate reader (Spectramax 384+, Molecular Devices, Sunnyvale, CA, USA). The concentration of mannitol in each sample was determined by preparing a standard curve of known concentrations. The concentration of mannitol in the RCC was used to calculate the volume of SAGM that had been delivered using the following equation:

$$
\mathrm{V}_{2}=\left(\mathrm{M}_{1} \times \mathrm{V}_{1}\right) / \mathrm{M}_{2}
$$

where $\mathrm{M}_{1}$ = RCC mannitol concentration (g/ml), $\mathrm{V}_{1=}$ RCC volume (ml), $\mathrm{M}_{2}$ $=$ SAGM mannitol concentration $(\mathrm{g} / \mathrm{ml}), \mathrm{V}_{2}=\mathrm{SAGM}$ volume $(\mathrm{ml})$.

\section{Albumin Measurements}

Albumin was measured in the samples using a bromocresol green colorimetric assay (BCG Albumin Assay Kit; Sigma-Aldrich). For both WBF- and BC-processed units, samples were taken from the final leukoreduced SAGM-RCC component, and also from the separated plasma component for measuring plasma albumin concentration. Plasma samples were diluted twofold with water. RCC samples were centrifuged at 2,200 $\times g$ for $10 \mathrm{~min}$ to separate the RBCs and supernatant, and the supernatant was removed. Samples and standards were added to albumin assay reagent in a 96-well plate and incubated at room temperature for $5 \mathrm{~min}$. Absorbance was read at $620 \mathrm{~nm}$ using a spectrophotometric plate reader (Spectramax 384+). The concentration of albumin in each sample was determined using a standard curve of known concentrations. The concentration of albumin was used to calculate the volume of plasma in the RCC using the following equation:

$$
\mathrm{V}_{2}=\left(\mathrm{A}_{1} \times \mathrm{V}_{1}\right) / \mathrm{A}_{2}
$$

where $\mathrm{A}_{1}$ = RCC albumin concentration $(\mathrm{g} / \mathrm{ml}), \mathrm{V}_{1}=\mathrm{RCC}$ volume $(\mathrm{ml}), \mathrm{A}_{2}=$ plasma albumin concentration $(\mathrm{g} / \mathrm{ml}) \mathrm{V}_{2}$ = residual plasma volume $(\mathrm{ml})$. 
Table 2. Mean volumes of SAGM and residual plasma in WBFand BC-processed RCC components*

\begin{tabular}{|c|c|c|c|c|c|}
\hline & \multirow[t]{2}{*}{ SAGM volume, $\mathrm{ml}^{\mathrm{a}}$} & \multicolumn{2}{|c|}{ Residual plasma volume, $\mathrm{ml}$} & \multirow[t]{2}{*}{ Residual plasma, $\%$} & \multirow[t]{2}{*}{ Ratio of plasma: SAGM } \\
\hline & & measured $(\mathrm{A})^{\mathrm{b}}$ & calculated $(\mathrm{B})^{\mathrm{c}}$ & & \\
\hline $\mathrm{WBF}(\mathrm{n}=9)$ & $105 \pm 2$ & $14 \pm 4$ & $5 \pm 3$ & $\begin{array}{l}\text { A: } 4.5 \pm 2.3 \\
\text { B: } 1.7 \pm 0.6\end{array}$ & $\begin{array}{l}\text { A: } 0.13 \pm 0.06 \\
\text { B: } 0.05 \pm 0.02\end{array}$ \\
\hline $\mathrm{BC}(\mathrm{n}=9)$ & $81 \pm 4$ & $11 \pm 3$ & $16 \pm 6$ & $\begin{array}{l}\text { A: } 4.3 \pm 1.3 \\
\text { B: } 6.1 \pm 0.6\end{array}$ & $\begin{array}{l}\text { A: } 0.14 \pm 0.04 \\
\text { B: } 0.20 \pm 0.03\end{array}$ \\
\hline \multicolumn{6}{|c|}{$\begin{array}{l}\text { a'SAGM volumes were calculated from experimental mannitol concentrations (Eq. 3). } \\
\text { bPlasma volume was calculated from experimental albumin measurements (Eq. 4). } \\
\text { 'Plasma volume was calculated using supernatant volume and SAGM volume (Eq. 1). } \\
\text { *Values shown are mean } \pm \text { SD. }\end{array}$} \\
\hline
\end{tabular}

\section{Results}

Supernatant volumes calculated using total unit volume and hematocrit (Eq. 2) were significantly lower in BC-processed units than in WBF units ( $p<0.05$; table 1$)$. The volume of SAGM was calculated using mannitol concentrations taken from the SAGM $(52.4 \pm 0.1 \mathrm{~g} / \mu \mathrm{l})$ and RCC samples $(\mathrm{BC}=16.6 \pm 0.04 \mathrm{~g} / \mu \mathrm{l}, \mathrm{WBF}=$ $17.1 \pm 0.04 \mathrm{~g} / \mu \mathrm{l}$; Eq. 3). Calculated SAGM volumes were significantly greater in WBF-processed RCCs compared to BC components $(\mathrm{p}<0.05$; table 2$)$, which is consistent with the difference in supernatant volumes.

Using albumin concentrations from plasma components and processed RCC components (plasma: $\mathrm{WBF}=4.46 \pm 0.40 \mathrm{~g} / \mathrm{dl}, \mathrm{BC}=$ $4.31 \pm 0.33 \mathrm{~g} / \mathrm{dl} ; \mathrm{RCCs}: \mathrm{WBF}=0.23 \pm 0.05 \mathrm{~g} / \mathrm{dl}, \mathrm{BC}=0.20 \pm 0.03$ $\mathrm{g} / \mathrm{dl}$ ), we were able to calculate the volume of plasma remaining in RCCs (Eq. 4). Residual plasma volumes calculated using Eq. 4 were similar in both WBF and BC components ( $\mathrm{p}=0.245$; table 2$)$. A calculated residual plasma volume (Eq. 1) was obtained using calculated supernatant volumes (table 1) and calculated SAGM volumes (table 2); showing a significant difference between the volume of plasma in both types of units $(\mathrm{p}<0.05)$.

\section{Discussion}

Previously, the volume of residual plasma has been calculated using supernatant volume and the volume of additive solution [1, 4]. When this calculation method was applied to a large-scale quality control database, supernatant values less than $110 \mathrm{ml}$ were observed, returning negative values for residual plasma volume. In this study we sought to more accurately calculate the volume of SAGM additive solution and residual plasma using mannitol and albumin concentrations.

We observed significantly lower supernatant volumes and additive solution volumes in $\mathrm{BC}$ components compared to $\mathrm{WBF}$ components. A key difference between the WBF- and BC-processing methods is the point of component leukoreduction (fig. 1). In the $\mathrm{BC}$ process, filtration occurs after component separation. The SAGM is passed through the filter to 'pre-wet' it before the SAGMRBCs are filtered back through. The filter may act as a sink for ad- ditive solution during the pre-wetting step, accounting for the reduced volume of SAGM observed in these components. The effect of additive solution volume on product quality has been examined [5], but there are no observations taking into account the actual volume of additive solution delivered to the RCC during processing. Previous studies have reported reduced storage hemolysis associated with larger volumes of additive solution, attributing increased buffer capacity as a possible reason [5].

Using the mannitol-based SAGM volume and RCC supernatant volume, we calculated significantly greater residual plasma volumes in BC-processed units compared to WBF units. This trend opposes the observations of previous groups $[1,4]$ that suggest that BC-RCCs have lower residual plasma volumes. We also observed disparity between residual plasma volume calculation methods; values calculated using albumin concentrations were higher than those calculated using Eq. 1 and were not influenced by the manufacturing method. One possible explanation for this is that the dispersion of the albumin (a large molecule) throughout the components is affected by centrifugation during separation. Collectively, this highlights the need for a standardized process for measuring residual plasma in RCCs.

The effect of RCC residual plasma on product characteristics and transfusion outcomes has not been fully investigated. Transfusion of plasma is associated with transfusion-related acute lung injury (TRALI) [6], the leading cause of transfusion-related deaths in North America. It has been suggested that larger volumes of residual plasma in RCCs could be associated with an increased risk of TRALI $[1,7]$. In addition to clinical outcomes, there is also interest in how residual plasma influences in vitro RCC quality during storage. In a recent study on neonatal RBCs stored in plasma, it was observed that a high plasma lipid content was associated with increased storage hemolysis [8].

The findings of this study emphasize that the supernatant in RCC products should not be viewed as a set volume of additive solution, but rather a variable mixture of plasma components and additive solution. Before we can begin speculating as to the in vitro implications or clinical significance of residual plasma in RCCs, we must first implement standardized, validated methods for measuring residual plasma volume. 


\section{Acknowledgements}

The authors would like to thank Canadian Blood Services and Health Canada for providing financial support, Dr. Geraldine Walsh, Canadian Blood Services Scientific Writer, for her assistance in manuscript preparation, and the netCAD staff and donors.

This research received financial support from Health Canada and Canadian Blood Services, funded by the provincial and territorial Ministries of Health.

\section{Disclosure Statement}

No conflicts of interest to disclose.

\section{References}

1 Weber LL, Roberts LD, Sweeney JD: Residual plasma in red blood cells and transfusion-related acute lung injury. Transfusion 2014;54:2425-2430.

2 Jordan A, Chen D, Yi QL, Kanias T, Gladwin MT, Acker JP: Assessing the influence of component processing and donor characteristics on quality of red cell concentrates using quality control data. Vox Sang 2015; (in press).

3 Acker JP, Hansen AL, Kurach JD, Turner TR, Croteau I, Jenkins C: A quality monitoring program for red blood cell components: in vitro quality indicators before and after implementation of semiautomated processing. Transfusion 2014;54:2534-2543.
4 de Grandmont M, Dion J, Dubuc S, Jacques A, Robillard P, Bedard C, Girard M, Thibault L: A simple approach for quality control monitoring of residual plasma in red blood cell concentrates. Transfusion 2014;54(suppl 2):70A.

5 Hess JR, Rugg N, Joines AD, Gormas JF, Pratt PG, Silberstein EB, Greenwalt TJ: Buffering and dilution in red blood cell storage. Transfusion 2006;46:50-54.

6 Bux J, Becker F, Seeger W: Transfusion-related acute lung injury due to HLA-A2-specific antibodies in recipient and NB1-specific antibodies in donor blood. $\mathrm{Br}$ J Haematol 1996;93:707-713.
7 Win N, Chapman CE, Bowles KM, Green A, Bradley S, Edmondson D, Wallis JP: How much residual plasma may cause TRALI? Transfus Med 2008;18:276-280.

8 Bashir S, Wiltshire M, Cardigan R, Thomas S: Lipaemic plasma induces haemolysis in resuspended red cell concentrate. Vox Sang 2013;104:218-224. 